\title{
Neoadjuvant chemotherapy in breast cancer: a dose-dense schedule in real life and putative role of PIK3CA mutations
}

\author{
Valentina Cocciolone ${ }^{1,2, *}$, Katia Cannita ${ }^{2, *}$, Alessandra Tessitore ${ }^{1}$, Valentina \\ Mastroiaco $^{1}$, Lucia Rinaldi ${ }^{2}$, Stefania Paradisi ${ }^{2}$, Azzurra Irelli ${ }^{2}$, Paola Lanfiuti \\ Baldi $^{2}$, Tina Sidoni ${ }^{2}$, Enrico Ricevuto ${ }^{1,3}$, Antonella Dal Mas ${ }^{4}$, Giuseppe Calvisi ${ }^{4}$, \\ Gino Coletti ${ }^{4}$, Antonietta Ciccozzi ${ }^{5}$, Laura Pizzorno ${ }^{6}$, Valter Resta ${ }^{6}$, Alberto Bafile ${ }^{6}$, \\ Edoardo Alesse ${ }^{1}$ and Corrado Ficorella ${ }^{1,2}$ \\ ${ }^{1}$ Department of Biotechnological and Applied Clinical Sciences, University of L'Aquila, L'Aquila, Italy \\ ${ }^{2}$ Medical Oncology Department, S. Salvatore Hospital, University of L'Aquila, L'Aquila, Italy \\ ${ }^{3}$ Oncology Network ASL1 Abruzzo, UOSD Oncology Territorial Care, S. Salvatore Hospital, University of L'Aquila, L'Aquila, \\ Italy \\ ${ }^{4}$ Pathology Department, S. Salvatore Hospital, L'Aquila, L'Aquila, Italy \\ ${ }^{5}$ Radiology Department, S. Salvatore Hospital, L'Aquila, L'Aquila, Italy \\ ${ }^{6}$ Breast Unit, S. Salvatore Hospital, L'Aquila, L'Aquila, Italy \\ "These authors have contributed equally to this work \\ Correspondence to: Valentina Cocciolone, email: vale.cocciolone@tiscali.it \\ Keywords: neaodjuvant; dose-dense; PIK3CA; real life \\ Received: July 27, $2017 \quad$ Accepted: April 06, $2018 \quad$ Published: June 08, 2018 \\ Copyright: Cocciolone et al. This is an open-access article distributed under the terms of the Creative Commons Attribution \\ License 3.0 (CC BY 3.0), which permits unrestricted use, distribution, and reproduction in any medium, provided the original author \\ and source are credited.
}

\section{ABSTRACT}

Background: Dose-dense chemotherapy is one of the treatments of choice for neoadjuvant therapy in breast cancer (BC). Activating mutations in PIK3CA gene predict worse response to neoadjuvant chemotherapy for HER2-positive patients, while their role is less clearly defined for HER2-negative tumors.

Methods: We conducted a phase I/II study of neoadjuvant, sequential, dosedense anthracycline/taxane chemotherapy, plus trastuzumab in HER2-positive patients and investigated the correlation of pre-treatment PIK3CA mutation status with pathologic complete response (PCR) and long-term outcome in a real-life setting.

Results: we established a dose-dense docetaxel recommended dose of $60 \mathrm{mg} / \mathrm{m}^{2}$ and $65 \mathrm{mg} / \mathrm{m}^{2}$, with or without trastuzumab, respectively, according to HER2-status, following dose-dense epirubicin-cyclophosphamide $\left(90 / 600 \mathrm{mg} / \mathrm{m}^{2}\right)$, every 2 weeks. The overall pCR rate was $\mathbf{2 1 . 4 \%}$; median disease-free survival (DFS) was 52 months and median overall survival (OS) was not yet reached. PIK3CA mutation status was not significantly associated with the PCR rate: $18 \%$ for both mutated and wild-type patients. The pCR rate was: $25 \%$ in the mutated and $24 \%$ in the wild-type ( $p 0.560)$ cohort of the HER2-positive subgroup; $33 \%$ both in the mutant and wild-type cohort of the triple-negative subgroup; no pCR neither in the mutant nor in the wild-type cohort of the HR-positive/HER2-negative subgroup. Among the HER2-positive population, a trend toward worse DFS was observed in case of mutation, as opposed to the triple negative population.

Conclusions: This study proposes an effective and safe neoadjuvant dose-dense anthracycline/taxane schedule and suggests that PIK3CA mutation analysis can be usefully performed in real-life clinical practice. 


\section{INTRODUCTION}

Neoadjuvant chemotherapy is considered the standard therapeutic approach to locally advanced breast cancer (LABC) and inflammatory breast cancer (IBC), but it should be considered the treatment of choice, in clinical practice, even in operable disease, based on the results of several randomized clinical trials showing its equivalence, in terms of DFS and OS, to adjuvant therapy [1]. Obtaining a pathologic complete response (pCR), defined as the absence of residual invasive carcinoma within both the breast and axillary lymph nodes after neoadjuvant treatment [2], has a significant impact on survival, enough to be considered a powerful surrogate marker [3-5]. Frequency of pCR in patients with lowgrade, hormone receptor (HR)-positive tumors is low, and more than doubled in the high-grade HR-positive tumors. Triple negative and human epidermal growth factor receptor 2 (HER2)-positive tumors are more likely able to achieve a pCR; within the HER2-positive population, $\mathrm{pCR}$ is more common for HR-negative than for HRpositive tumors, and with the addition of trastuzumab. The most favorable outcomes after $\mathrm{pCR}$ are detected in patients with HER2-positive/HR-negative tumors who receive trastuzumab, and in the triple-negative subgroup [6]. The results of the B-27 trial showed an $87 \%$ and a $16 \%$ increase in pCR rate and in negative axillary nodes, respectively, with the sequential addition of preoperative docetaxel after completion of preoperative doxorubicincyclophosphamide. The addition of trastuzumab to neoadjuvant chemotherapy in the HER2-positive disease significantly increased the $\mathrm{pCR}$ rate compared to chemotherapy alone $[7,8]$, with a favorable impact on the event-free survival, strongly associated with $\mathrm{pCR}$ only in patients treated with trastuzumab [9]. Based on the Gompertzian kinetic model of cancer growth [10], increasing dose density improves outcomes [11] by killing more cancer cells as they re-grow after the previous cycle of therapy, making reasonable to hypothesize that shortening the interval between cycles of chemotherapy may be more critical in high-grade rapidly proliferating tumors [12]. A meta-analysis of randomized controlled trials, comparing dose-dense (dd) chemotherapy with a conventional schedule in non-metastatic $\mathrm{BC}$, showed that the dose-dense approach results in better OS and DFS, particularly in women with HR-negative disease, even if it was associated with a greater incidence of nonhematological adverse events [13].

Therefore, we conducted a phase I/II study of neoadjuvant dd anthracycline/taxane chemotherapy (plus trastuzumab (T) in HER2-positive disease) in BC patients, planned to define the recommended dose (RD) of dd Docetaxel (D) following dd Epirubicin/Cyclophosphamide (EC).

In recent years, increasing importance is gaining the evaluation of the phosphatidylinositol-3- kinase (PI3K) mutational status as a prognostic and predictive factor. Mutations of the p110 $\alpha$ catalytic domain of PI3K, encoded by the PIK3CA gene, are the most common genetic alterations of the PI3K/Akt/ mammalian target of rapamycin (mTOR) pathway in $\mathrm{BC}$, being identified in approximately $20 \%$ of all BCs. About $80 \%$ of them occur at the level of the $\alpha$-helical (Exon 9) and kinase domain (Exon 20) of the p110 $\alpha$ subunit, involving three hotspot sites [14]: a hotspot in the kinase domain, with the aminoacidic substitution of H1047R, inducing a conformational change in the protein activation loop, responsible for enzymatic hyperactivity; two other hotspots in the helical domain, consisting of the E542K and E545K substitutions, resulting in ineffective regulation of the $\mathrm{p} 110 \alpha$ kinase activity, mediated by the regulatory subunit. In BC, mutations in Exon 20 are more frequent than in Exon 9 [15]. However, the frequency of PIK3CA mutations is not equally distributed among the different biologic subtypes [16]: a gene mutation has been reported up to $45 \%$ in Luminal-A BCs , in $29 \%$ of Luminal-B and in $39 \%$ of HER 2 -positive tumors, while approximately $9 \%$ of basal-like tumors harbor a PIK3CA mutation.

In the neoadjuvant setting, PIK3CA mutations do not appear to be associated with altered sensitivity to preoperative chemotherapy with anthracyclines and taxanes, although mutations in exon 9 are associated with a higher rate of node-negative residual disease, especially in the subgroup of patients with estrogen receptor (ER)positive BC [17]. Analyses from patients with HER2+ BC treated with neoadjuvant anthracyclines, taxanes and antiHER2 agents, showed that activating mutations in PIK3CA predict a lower chance of achieving a pCR, while they did not significantly impact DFS and OS $[18,19]$. The negative prognostic impact of PIK3CA mutations in $\mathrm{BC}$ is more evident in the metastatic disease: worse median progression-free survival was observed for patients whose tumors expressed mutated versus wild-type PIK3CA, in both the control (8.6 versus 13.8 months) and pertuzumab groups (12.5 versus 21.8 months) [20].

In the light of these premises, in this study we also assessed the correlation of pre-treatment PIK3CA mutation status with pCR, relapse-free survival (RFS), DFS and OS of enrolled patients, according to the histological subgroups.

\section{RESULTS}

\section{Baseline characteristics}

From October 2010 to August 2015, 42 consecutive, unselected patients were enrolled (Table 1), 12 in the phase I part of the study. Median age was 50 years (range $38-66)$. As to disease extension, 19 patients $(45.5 \%)$ had operable stage II-IIIA BC, $22(52.5 \%)$ LABC and 1 patient $(2 \%)$ IBC. Out of 44 evaluable tumors (2 patients had 


\begin{tabular}{|c|c|}
\hline & N. $(\%)$ \\
\hline N. patients & 42 \\
\hline Age, years median & 50 \\
\hline range & $38-66$ \\
\hline \multicolumn{2}{|l|}{ Menopause Status } \\
\hline Pre & $28(67)$ \\
\hline Post & $14(33)$ \\
\hline \multicolumn{2}{|l|}{ WHO Performance Status } \\
\hline 0 & $40(95)$ \\
\hline 1 & $2(5)$ \\
\hline \multicolumn{2}{|l|}{ CIRS } \\
\hline Primary & $27(64)$ \\
\hline Intermediate & $12(29)$ \\
\hline Secondary & $3(7)$ \\
\hline \multicolumn{2}{|l|}{ Histological type } \\
\hline Ductal & $36(86)^{*}$ \\
\hline Lobular & $5(12)^{*}$ \\
\hline $\begin{array}{l}\text { Other } \\
*(2 \text { pts with bilateral breast cancer })\end{array}$ & $3(7)$ \\
\hline \multicolumn{2}{|l|}{ Stage of disease } \\
\hline Operable EBC & $19(45.5)$ \\
\hline LABC & $22(52.5)$ \\
\hline IBC & $1(2)$ \\
\hline \multicolumn{2}{|l|}{ Cardiovascular comorbidity } \\
\hline Yes & $9(21)$ \\
\hline No & $33(79)$ \\
\hline
\end{tabular}

bilateral carcinoma), 54.5\% were HER2-positive (ER+/ Progesterone Receptor (PgR)+, 16\%; ER+/PgR-, 13.5\%; ER-/PgR-, 25\%) and 45.5\% HER2-negative (ER+/PgR+, 18.5\%; ER+/PgR-, 13.5\%; ER-/PgR-, 13.5\%).

\section{Dose-finding}

Overall, 5 dose-limiting toxicities (DLTs) were observed out of 12 patients (42\%). Among HER2-positive patients, DLTs occurred in all 3 patients enrolled in the first cohort at D $65 \mathrm{mg} / \mathrm{m}^{2}$, represented by G3 asthenia in the first 2 patients, occurred at the second and fourth cycle, respectively, and G2 asthenia lasting more than 2 weeks in the third patient, occurred at the first cycle. Therefore, a next cohort of 3 patients was necessarily enrolled at D $60 \mathrm{mg} / \mathrm{m}^{2}$; at this dose-level, no DLT was reported. Thus, $60 \mathrm{mg} / \mathrm{m}^{2}$ was established as D RD in combination with
T. Among HER2-negative patients, DLT, represented by G3 hand-foot syndrome (HFS), occurred in 1 out of 3 patients enrolled in the first cohort at D $65 \mathrm{mg} / \mathrm{m}^{2}$, so a new cohort was enrolled at the same dose-level and 1 DLT, represented by G2 anemia for more than 2 weeks, occurred. Thus, $65 \mathrm{mg} / \mathrm{m}^{2}$ was D RD when administered alone.

\section{Treatment administration}

Out of 42 enrolled pts, 38 (90\%) completed the treatment cycles planned in the study (4 cycles of EC and 5-6 cycles of $\mathrm{D}$ with/without $\mathrm{T}$ ), as two patients, 1 of the HER2-positive and 1 of the HER2-negative group, reported a hypersensitivity reaction to $\mathrm{D}$ at the second administration and continued treatment with nanoparticle albumin-bound (nab)-Paclitaxel and two patients, in the 
HER2-negative subgroup, refused to undergo the last D cycle to anticipate surgery. The two patients who had the hypersensitivity reaction to D were not included in the D safety analysis. The total number of administered cycles was 395 (168 cycles of EC, 128 cycles of D with T and 99 cycles of D alone). Overall, median absolute dose intensity (DI) of E was $45 \mathrm{mg} / \mathrm{m}^{2} /$ week (range 33.3-45); median absolute DI of D in HER2-negative and HER2-positive population was $30.5 \mathrm{mg} / \mathrm{m}^{2} /$ week (range $23.5-32.5$ ) and $29.75 \mathrm{mg} / \mathrm{m}^{2} /$ week (range $24.2-30$ ), respectively.

\section{Activity and efficacy}

Activity and efficacy data are shown in Table 2. In the intention-to-treat (ITT) analysis, all 42 enrolled patients were evaluable for activity: pCR was obtained in 8 patients $(19 \%)$. In the as-treated analysis, 40 patients were evaluable, as two patients reported a hypersensitivity reaction to $\mathrm{D}$ at the second administration and continued treatment with nab-Paclitaxel: pCR was achieved in 8 patients $(20 \%)$. As one of the two patients treated with nab-Paclitaxel obtained a $\mathrm{pCR}$, the $\mathrm{pCR}$ rate in the whole population treated with sequential EC and taxanes was $21.4 \%$ (9 out of 42 patients). Four of these pCRs were characterized by residual carcinoma in situ in the breast with negative nodes. As two patients had a synchronous bilateral BC, the subgroup activity analysis was performed on 42 tumors treated with $\mathrm{EC} \rightarrow \mathrm{D} \pm \mathrm{T}$ and 44 tumors treated with $\mathrm{EC} \rightarrow$ Taxane $\pm \mathrm{T}$. In the as-treated analysis (42 tumors), in the HER2-positive population, treated with $\mathrm{D}$ in combination with $\mathrm{T}$, pCR was observed in 6 out of 23 tumors (26\%); 3 pCRs were reported out of 13 ER-positive tumors (23\%) and 3 out of 10 ER-negative tumors $(30 \%)$. In the HER2-negative population, pCR was observed in 3 out of 19 tumors (15.7\%); 2 pCRs were reported out of 6 triple-negative tumors (TNBC) (33.3\%) and 1 out of 13 ER-positive/HER2-negative tumors (7.7\%) (Table 3a). As the patient treated with nab-Paclitaxel who reported the $\mathrm{pCR}$ had a HR-negative/HER2-positive tumor, in the ITT analysis of patients treated with EC $\rightarrow$ Taxane $\pm \mathrm{T}$ (44 tumors), pCR was observed in 7 out of 24 tumors $(29.2 \%)$ in the HER2-positive population: 3 pCRs were reported out of 13 ER-positive tumors (23\%) and 4 out of 11 ER-negative tumors (36.4\%). In the HER2-negative population, pCR was observed in 3 out of 20 tumors (15\%); 2 pCRs were reported out of 6 triplenegative tumors $(33.3 \%)$ and 1 out of 14 ER-positive/ HER2-negative tumors (7.1\%) (Table $3 \mathrm{~b}$ ). After a median follow-up of 33 months for both ITT and as-treated analysis, overall median RFS and DFS were 57 months and 52 months, respectively: 16 patients underwent distant disease recurrence ( 5 on brain, 2 on liver, 1 on lung and pleura and 4 on lymph nodes) and 2 patients had a local recurrence on the breast (including cutaneous diffusion and regional lymph nodes involvement); 1 patient had a second tumor on esophagus. Median RFS was not reached, 57 months and 12 months for the HER2+, HR+/
HER2- and triple-negative subgroup, respectively; median DFS was not reached, 52 months and 7.5 months for the HER2+, HR+/HER2- and triple-negative subgroup, respectively (Table 4). Out of 23 HER2-positive patients, 7 $(30 \%)$ disease recurrences occurred [3 out of 11 ER-/PgR-/ HER $2+(27 \%)$ and 4 out of $12 \mathrm{ER}+/ \mathrm{PgR}+$ or -/HER2+ $(33 \%)]$. Out of 6 triple-negative patients, 4 (67\%) disease recurrences occurred, and one patient had the esophageal tumor. Out of $13 \mathrm{ER}+/ \mathrm{PgR}+$ or -/HER2-negative patients, $5(38 \%)$ disease recurrences occurred [3 out of $7 \mathrm{ER}+/$ PgR+/HER2- (43\%), and 2 out of 6 ER+/PgR-/HER2$(33 \%)]$. Overall median OS was not reached: 10 patients died due to $\mathrm{BC}, 1$ patient died due to esophageal tumor. Median OS was not reached for the HER2+ and HR+/ HER2- subgroup and 29.5 months for the triple-negative subgroup. Ten patients died due to BC: HER2-positive, 3; triple-negative, 4; HR+/HER2-negative, 3; 1 patient with TNBC died due to esophageal tumor.

\section{Toxicity}

All patients were evaluated for EC toxicity, 22 patients for $\mathrm{D}$ in combination with $\mathrm{T}$ and 18 for $\mathrm{D}$ alone toxicity. During the EC phase, regarding nonhematological toxicity, asthenia was the most common severe toxicity ( $7 \%$ of patients, $4 \%$ of cycles). The incidence of other G3 non-hematological toxicities was rare, and included only vomiting and transaminases increase in $2 \%$ of patients, $0.5 \%$ of cycles. The single severe hematological toxicity was neutropenia (with or without leucopenia), which occurred in $40 \%$ of patients (14\% G3 and 26\% G4) and $15 \%$ of cycles. Febrile neutropenia was not reported (Supplementary Table 1). During the $D$ phase, a distinction must be done between the HER2-positive group, treated with D 60 $\mathrm{mg} / \mathrm{m}^{2}$ in combination with $\mathrm{T}$, and the HER2-negative group, treated with D $65 \mathrm{mg} / \mathrm{m}^{2}$ alone. Regarding non-hematological toxicity, asthenia was once again the most frequent toxicity, occurring at G3 in 18\% of patients and $3 \%$ of cycles, and in $11 \%$ of patients and $2 \%$ of cycles in the HER2-positive and HER2-negative group, respectively. Anyway, G2 asthenia was more frequent in the HER2-negative group (56\% versus $3 \%$ of patients). Severe transaminases increase was reported by 1 patient $(5 \%)$ in 1 cycle $(0.7 \%)$ in the HER2-positive group, while severe myalgia occurred only in the HER2-negative group, by 1 patient $(6 \%)$ in 1 cycle $(2 \%)$. As to hematological toxicity, G3-4 neutropenia (with or without leucopenia) occurred in $12 \%$ of patients $(6 \% \mathrm{G} 3$ and $6 \% \mathrm{G} 4)$ in $3 \%$ of cycles, only in the D alone arm (Supplementary Table 2). Cumulatively, cardiac toxicity was reported in 28 out of all 42 enrolled patients (67\%) and 39 cycles (10\%), with no limiting events, thus represented only by cardiac dysfunctions, not requiring treatment delay or interruption (Supplementary Table 3a). Neither c-TnI nor precursor-Brain-Natriuretic-Peptide (pBNP) increase was 
Table 2: Activity and efficacy

\begin{tabular}{|c|c|c|c|c|c|}
\hline & & \multicolumn{2}{|c|}{ Intention-to-treat analysis } & \multicolumn{2}{|c|}{ As-treated analysis } \\
\hline & & N. & $\%$ & N. & $\%$ \\
\hline \multirow[t]{3}{*}{$\operatorname{ddEC} \rightarrow \mathrm{D} \pm \mathrm{T}$} & Enrolled patients & 42 & 100 & 42 & 100 \\
\hline & $\begin{array}{c}\text { Evaluable } \\
\text { patients }\end{array}$ & 42 & 100 & 40 & 95 \\
\hline & $\mathbf{p C R}$ & 8 & 19 & 8 & 20 \\
\hline \multirow[t]{3}{*}{$\begin{aligned} \text { ddEC } & \rightarrow \text { Taxane } \\
& \pm T\end{aligned}$} & Enrolled patients & 42 & 100 & 42 & 100 \\
\hline & $\begin{array}{c}\text { Evaluable } \\
\text { patients }\end{array}$ & 42 & 100 & 42 & 100 \\
\hline & pCR & 9 & 21.4 & 9 & 21.4 \\
\hline \multicolumn{2}{|c|}{ Median RFS, months } & \multicolumn{2}{|c|}{$57 \mathrm{mo}$} & & \\
\hline \multicolumn{2}{|l|}{ Range } & \multicolumn{2}{|c|}{$7-65+$} & & \\
\hline \multicolumn{2}{|l|}{ Progression events } & \multicolumn{2}{|c|}{$16(+1)^{*}$} & & \\
\hline \multicolumn{2}{|c|}{ Median DFS, months } & \multicolumn{2}{|c|}{$52 \mathrm{mo}$} & & \\
\hline \multicolumn{2}{|l|}{ Range } & \multicolumn{2}{|c|}{$2-58+$} & & \\
\hline \multicolumn{2}{|l|}{ Progression events } & \multicolumn{2}{|c|}{$16(+1)^{*}$} & & \\
\hline \multicolumn{2}{|c|}{ Median OS, months } & \multicolumn{2}{|c|}{$\mathrm{nr}$} & & \\
\hline \multicolumn{2}{|l|}{ Range } & \multicolumn{2}{|c|}{$9-71+$} & & \\
\hline \multicolumn{2}{|l|}{ Deaths } & \multicolumn{2}{|c|}{$10(+1)^{*}$} & & \\
\hline
\end{tabular}

* 1 patient had a second tumor on esophagus and died because of it.

Table 3a: pCR according to BC subtype on as-treated 42 evaluable tumors

\begin{tabular}{llcr}
\hline & & \multicolumn{2}{c}{ HER2 } \\
\cline { 3 - 4 } & & positive & Negative \\
\cline { 3 - 4 } ER & & D+T & D alone \\
& negative & $3 / 10(30 \%)$ & $2 / 6(33.3 \%)$ \\
& positive & $3 / 13(23 \%)$ & $1 / 13(7.7 \%)$ \\
\hline
\end{tabular}

Table 3b: pCR according to BC subtype on ITT 44 evaluable tumors

\begin{tabular}{llcc}
\hline & & \multicolumn{2}{c}{ HER2 } \\
\cline { 3 - 4 } & & positive & Negative \\
\cline { 3 - 4 } ER & & D+T & D alone \\
& negative & $4 / 11(36.4 \%)$ & $2 / 6(33.3 \%)$ \\
& positive & $3 / 13(23 \%)$ & $1 / 14(7.1 \%)$ \\
& Overall & $7 / 24(29.2 \%)$ & $3 / 20(15 \%)$ \\
\hline
\end{tabular}


Table 4: Efficacy according to subtypes

\begin{tabular}{lccc}
\hline & HER2 + & HR+/HER2 - & TNBC \\
\hline Median RFS, months & $\mathrm{nr}$ & 57 mo & $12 \mathrm{mo}$ \\
Progression events & 7 & 5 & $4(+1)^{*}$ \\
Median DFS, months & $\mathrm{nr}$ & $52 \mathrm{mo}$ & $7.5 \mathrm{mo}$ \\
Progression events & 7 & 5 & $4(+1)^{*}$ \\
Median OS, months & $\mathrm{nr}$ & $\mathrm{nr}$ & $29.5 \mathrm{mo}$ \\
Deaths & 3 & 3 & $4(+1)^{*}$ \\
\hline
\end{tabular}

* 1 patient had a second tumour on esophagus and died because of it.

reported. Two patients, one with HER2-positive and one with HER2-ngative tumor, had an asymptomatic $\leq 10 \%$ left ventricular ejection fraction (LVEF) reduction from baseline with a final value, at treatment completion, of $50 \%$. The mean overall LVEF baseline value was $65 \%$, maintained after the first treatment phase with EC (64\%) and up to chemotherapy completion (65\%). In the HER2positive population, median LVEF was $65 \%, 64 \%$ and $61 \%$ at baseline, after EC and at treatment completion, respectively; in the HER2-negative population, median LVEF was maintained at $65 \%$ in all three evaluations (Supplementary Table 3b).

\section{Frequency of $P I K 3 C A$ mutation and association with clinicopathological factors}

Thirty-nine out of 42 tumor samples $(92.8 \%)$ were analyzed for exon 9 and 20 PI3KCA mutations. Mutations of exon 9 were more common $(9 / 39,23 \%)$ than mutations of exon $20(1 / 39,3 \%)$; one of the tumors $(3 \%)$ harbored a mutation in both exons. Mutations detected in exon 9 were c. $1624 \mathrm{G}>\mathrm{A}$ (corresponding to the E542K amino acid substitution) alone in 8 cases, associated with c. $1633 \mathrm{G}>\mathrm{A}$ (corresponding to the $\mathrm{E} 545 \mathrm{~K}$ amino acid substitution) in 2 cases; mutations detected in exon 20 were c. $3140 \mathrm{~A}>\mathrm{G}$ (corresponding to the H1047R amino acid substitution) in 1 case and c.3129G $>$ A (corresponding to the M1043I amino acid substitution) in 1 case (Table 5) (Figure 1). The latter mutation was described as very infrequent in the Cosmic database (http://cancer.sanger.ac.uk/). No significant correlation between $P I K 3 C A$ mutation status and other baseline characteristics was observed. A PIK3CA mutation was detected in 4/21 (19\%) HER2-positive tumors, 3/6 (50\%) triple-negative tumors and 4/12 (33\%) HR-positive/HER2-negative tumors, with not statistically significant differences between subgroups ( $p$ 0.296).

\section{Association of PIK3CA mutations and response to treatment}

The presence of a PIK3CA mutation, overall, was not significantly associated with the pCR rate: 2 out of 11
(18\%) patients with $P I K 3 C A$-mutated tumors achieved a pCR compared to 5 out of 28 (18\%) patients in the wildtype cohort (odds ratio (OR), $1.022 ; 95 \% \mathrm{CI}, 0.167$ to 6.261 ; p 0.981). Of note, the tumor tissue of 2 patients achieving pCR was not available for PIK3CA mutational analysis. By subgroups, in the HER2-positive population, the pCR rate was $25 \%(1 / 4)$ in the mutated cohort and $23.5 \%(4 / 17)$ in the wild-type cohort (OR, 1.083; 95\% CI, 0.087 to 13.55 ; p 0.950$)$; in the triple-negative population, it was $33 \%$ both in the mutated $(1 / 3)$ and in the wild-type cohort (1/3) (OR, 1.000; 95\% CI, 0.033 to 29.83; p 1.000); in the HR-positive/HER2-negative population, no pCR was obtained neither in the mutated $(0 / 4)$ nor in the wildtype $(0 / 8)$ cohort. Within the HER2-positive subgroup, the pCR rate was 33\% (3/9) for the HR-negative and PIK3CA wild-type cohort and 50\% (1/2) for the HR-negative and PIK3CA mutated cohort (OR, 0.5; 95\% CI, 0.023 to 11.10 ; p 0.658$) ; 12.5 \%$ (1/8) for the HR-positive and PIK3CA wild-type cohort, while any patient in the HR-positive and PIK3CA mutated cohort achieved the pCR (OR, $1.000 ; 95 \%$ CI, 0.030 to 33.35 ; p 0.598). The response to treatment according to the Residual Cancer Burden (RCB) score was not significantly related to the $P I K 3 C A$ mutational status, as the frequency of $P I K 3 C A$ mutations was similar in patients with pCR, RCB-I, RCB-II or RCBIII (p 0.454) (Table 6).

\section{Association between $P I K 3 C A$ genotype and long- term survival}

Overall, neither DFS nor OS were statistically significantly different between patients with or without a PIK3CA mutation: median DFS was not reached in the mutated cohort versus 52 months in the wild-type cohort (hazard ratio, $1.185 ; 95 \% \mathrm{CI}, 0.396$ to $3.541 ; \mathrm{p} 0.761$ ); median OS was not reached in both the mutated and wildtype cohort (hazard ratio, $0.933 ; 95 \% \mathrm{CI}, 0.250$ to 3.478 ; $\mathrm{p}$ 0.918). Within the HER2-positive subgroup, median DFS was 23 months in the mutated cohort versus not reached in the wild-type cohort (hazard ratio, 2.731; 95\% CI, 0.350 to 21.28 ; $p$ 0.337); median OS was not reached in both the mutated and wild-type cohort (hazard ratio, 0.285; 95\% 
Table 5: Characteristics of patients carrying PIK3CA mutations

\begin{tabular}{|c|c|c|c|c|c|c|c|c|}
\hline & RE/RPg & HER2 & Activity & DFS & RFS & OS & PIK3CA ex9 & PIK3CA ex20 \\
\hline \multicolumn{9}{|c|}{ HER2-positive } \\
\hline AQ01 & $95 / 40$ & pos & $\begin{array}{c}\text { ypT2 } \\
\text { ypN3a }\end{array}$ & 11 & 17 & $25+$ & c. $1624 \mathrm{G}>\mathrm{A}$ & wt \\
\hline AQ02 & $95 / 95$ & pos & $\begin{array}{l}\text { ypT1b } \\
\text { ypN0 }\end{array}$ & $21+$ & $27+$ & $27+$ & wt & c. $3129 \mathrm{G}>\mathrm{A}$ \\
\hline AQ03 & neg/neg & pos & $\mathrm{pCR}$ & $35+$ & $41+$ & $41+$ & $\begin{array}{l}\text { c. } 1624 \mathrm{G}>\mathrm{A} \\
\text { c. } 1633 \mathrm{G}>\mathrm{A}\end{array}$ & wt \\
\hline AQ04 & neg/neg & pos & $\begin{array}{l}\text { ypT1c } \\
\text { ypN2 }\end{array}$ & 2 & 7 & $43+$ & c. $1624 \mathrm{G}>\mathrm{A}$ & wt \\
\hline \multicolumn{9}{|c|}{ TNBC } \\
\hline AQ05 & neg/neg & neg & $\mathrm{pCR}$ & $29+$ & $36+$ & $36+$ & $\begin{array}{l}\text { c. } 1624 \mathrm{G}>\mathrm{A} \\
\text { c. } 1633 \mathrm{G}>\mathrm{A}\end{array}$ & wt \\
\hline AQ06 & neg/neg & neg & $\begin{array}{l}\text { ypT1c } \\
\text { ypN0 }\end{array}$ & 26 & 31 & 33 & c. $1624 \mathrm{G}>\mathrm{A}$ & wt \\
\hline AQ07 & neg/neg & neg & $\begin{array}{l}\text { ypT1a } \\
\text { ypN0 }\end{array}$ & 6 & 10 & 26 & c. $1624 \mathrm{G}>\mathrm{A}$ & wt \\
\hline \multicolumn{9}{|c|}{ HR+/HER2- } \\
\hline AQ08 & 30/neg & neg & ypTx ypN1 & $47+$ & $54+$ & $54+$ & c. $1624 \mathrm{G}>\mathrm{A}$ & c. $3140 \mathrm{~A}>\mathrm{G}$ \\
\hline AQ09 & $65 / 5$ & neg & $\begin{array}{l}\text { ypT1c } \\
\text { ypN0 }\end{array}$ & $28+$ & $33+$ & $33+$ & c. $1624 \mathrm{G}>\mathrm{A}$ & wt \\
\hline AQ10 & $80 / 1$ & neg & урT3 урN3 & 3 & 7 & 19 & c. $1624 \mathrm{G}>\mathrm{A}$ & wt \\
\hline AQ11 & $70 / 60$ & neg & $\begin{array}{l}\text { ypT1a } \\
\text { ypN0 }\end{array}$ & $60+$ & $65+$ & $65+$ & c. $1624 \mathrm{G}>\mathrm{A}$ & wt \\
\hline
\end{tabular}

c. $1624 \mathrm{G}>\mathrm{A}$ corresponds to $\mathrm{E} 542 \mathrm{~K}$; c. $1633 \mathrm{G}>\mathrm{A}$ corresponds to $\mathrm{E} 545 \mathrm{~K}$; c.3140A $>\mathrm{G}$ corresponds to H1047R; c.3129G $>$ A corresponds to M1043I.

CI, 0.016 to $4.940 ; \mathrm{p} 0.389)$. In this subgroup, similar results were obtained when analyzing data based on HR status: within the HR-positive/HER2-positive population, median DFS was 16 months in the mutated cohort versus not reached in the wild-type cohort (hazard ratio, 2.784; 95\% CI, 0.146 to 53.05; p 0.496); median OS was not reached in both the mutated and wild-type cohort (hazard ratio, $0.286 ; 95 \% \mathrm{CI}, 0.0021$ to 38.47 ; $\mathrm{p} 0.617$ ); within the HR-negative/HER2-positive population, median DFS was 18.5 months in the mutated cohort versus not reached in the wild-type cohort (hazard ratio, 6.041; 95\% CI, 0.207 to 176.3 ; p 0.296); median OS was not reached in both the mutated and wild-type cohort (hazard ratio, 0.263; 95\% CI, 0.0028 to 24.36; p 0.563). Differences in outcome between HR-negative/HER2-positive mutated cohort and HR-positive/HER2-positive mutated cohort were not significantly different (DFS: hazard ratio, 1.423; 95\% CI, 0.082 to 24.66 ; p 0.808 . OS: any event of death). Within the triple-negative subgroup, median DFS was 26 months versus 3 months in the mutated and wild-type cohort, respectively (hazard ratio, $1.173 ; 95 \% \mathrm{CI}, 0.019$ to 1.53 ; p 0.115); median OS was 33 months versus 19 months in the mutated and wild-type cohort, respectively (hazard ratio, $0.563 ; 95 \% \mathrm{CI}, 0.071$ to $4.446 ; \mathrm{p} 0.586$ ). Within the HR-positive/HER2-negative subgroup, median DFS was not reached in the mutated cohort versus 52 months in the wild-type cohort (hazard ratio, $0.631 ; 95 \% \mathrm{CI}, 0.091$ to 4.34 ; p 0.640); median OS was not reached in both the mutated and wild-type cohort (hazard ratio, 1.005; 95\% CI, 0.09 to 11.14 ; p 0.997) (Table 7). Differences in outcome between HR-positive/HER2-negative mutated cohort and HR-positive/HER2-positive mutated cohort were not significantly different (DFS: hazard ratio, 0.561 ; 95\% CI, 0.031 to 10.03 ; p 0.694; OS: hazard ratio, 4.482; $95 \%$ CI, 0.070 to 286.5; p 0.479). Kaplan-Meier curves of DFS are shown in Figure 2.

\section{DISCUSSION}

The present phase I-II study proposes a sequential regimen of dose-dense EC followed by dose-dense D 
with or without $\mathrm{T}$, according to HER2-status, at D RD of $60 \mathrm{mg} / \mathrm{m}^{2}$ and $65 \mathrm{mg} / \mathrm{m}^{2}$, respectively, over a 20-weeks period before surgery. The dose-dense approach allows to safely administer D at a median DI of $29.75 \mathrm{mg} / \mathrm{m}^{2} /$ week in the HER2-positive population and $30.5 \mathrm{mg} / \mathrm{m}^{2} /$ week in the HER2-negative one, by improving the toxicity profile. The maintenance of an adequate DI was the limit of several studies testing, in the neoadjuvant setting, the sequence or combination of anthracyclines and taxanes in a dose-dense schedule: they could not demonstrate the feasibility, in clinical practice, of D at $100 \mathrm{mg} / \mathrm{m}^{2}$ every 2 weeks [21, 22], except when planning an inversion of the sequence of drugs, that is administering taxanes before the anthracycline [23].

As we conducted a monocentric study, achieving the sample size planned to complete the enrollment required for the phase II, takes too much time. Therefore, the enrollment has been closed in advance. Anyway, in the phase I of the study we identified the recommended doses of docetaxel in the two different subgroups of patients and the preliminary data of the phase II analysis show that the proposed regimen produces results that are consistent with those of the major and larger studies of sequential anthracyclines and taxanes in alternative schedules. In fact, the overall 19\% pCR rate obtained in the present study with $\mathrm{EC} \rightarrow \mathrm{D}$ and $21.4 \%$ in the overall study with sequential anthracycline and taxane, is comparable to $17.1 \%$ achieved by the sequential regimen dd EC followed by D plus capecitabine we previously reported [24] and to those achieved in the largest 3 -weekly anthracyclines-taxanes sequential trials. In the NSABP B-27, pCR on breast and axillary nodes (absence of invasive cancer cells) was obtained in $21.8 \%$ of patients treated with 4 cycles of AC $\left(60 / 600 \mathrm{mg} / \mathrm{m}^{2}\right)$ every 21 days, followed by 4 cycles of $\mathrm{D}\left(100 \mathrm{mg} / \mathrm{m}^{2}\right)$ every 21 days (group II). Anyway, the cumulative treatment completion rate was $80.7 \%$ (78.8\% in group II) and $10.9 \%$ of patients discontinued D because of adverse events [25]. In the Her2-positive subgroup, we obtained a pCR rate of $26 \%$ if considering $\mathrm{EC} \rightarrow \mathrm{D}$ and $29.2 \%$ if considering the overall population treated with sequential anthracycline and taxane. Even if this pCR rate is lower than the 38\% expected according to the meta-analysis by Valachis et al [26], it should be noted that it included studies where trastuzumab was administered upfront, concomitantly with both anthracyclines and taxanes, where efficacy seemed to be better compared to studies not combining anthracyclines and trastuzumab. Anyway, in the study by Pierga et al [27], where patients received EC (75/750 $\left.\mathrm{mg} / \mathrm{m}^{2}\right)$ every 3 weeks for 4 cycles followed by D (100 $\mathrm{mg} / \mathrm{m}^{2}$ ) every 3 weeks for 4 cycles with trastuzumab, $26 \%$ had a pCR (no residual invasive cells on breast and nodes), with a good toxicity profile and no cardiac event. Our pCR rate, comparable to this, was obtained in a shorter period, and with any toxicity increase. The higher pCR rate in the HR-/HER2+ subgroup, compared to the HR+/HER2+ subgroup (30\% vs 23\% with EC $\rightarrow$ D and $36.4 \%$ vs $25 \%$ with sequential anthracycline and taxane), is in line with literature data. With the 3-weekly schedule just mentioned, $32 \%$ of patients in the ER-/PgR-group and $20.5 \%$ in the ER+ and/or PgR + group achieved a pCR [27]; in the TECHNO study, the pCR rates were $42.3 \%$ and $35.4 \%$ for patients in the HR- and HR+ populations, respectively [28]; Buzdar al reported a $70.4 \%$ of pCR rate in women with ER-/PgR- BC versus $47.6 \%$ in women with $\mathrm{ER}+$ or $\mathrm{PgR}+$ disease, with trastuzumab administered with paclitaxel following FEC [29]. Since, so far, there are no studies investigating $\mathrm{D}$ in combination with trastuzumab in a dose-dense schedule, we cannot know how much our regimen could be improved, unless hypothesizing the introduction of a dual HER2-blockade, that showed high efficacy compared to trastuzumab alone [30-34]. In the triple-negative population, we obtained a pCR rate of $33.3 \%$, comparable to those obtained, in this subset, with sequential anthracyclines-taxanes regimens $[25,27,35,36]$ and to the meta-analytic results by Houssami et al [37]. There are potential reasons to believe that the benefits of dose-dense therapy could be particularly effective for specific subtypes such as triple-negative tumors, biologically characterized by a high proliferation rate and constitutive expression of genes related to proliferation [38]. On the other hand, TNBC is a highly diverse group of cancers and further sub-classification is needed for predicting the benefits of chemotherapy [39]. The challenge is therefore to identify subsets of patients with high probability of achieving a $\mathrm{pCR}$, allowing them to have a good long-term prognosis after neoadjuvant chemotherapy, considering the high

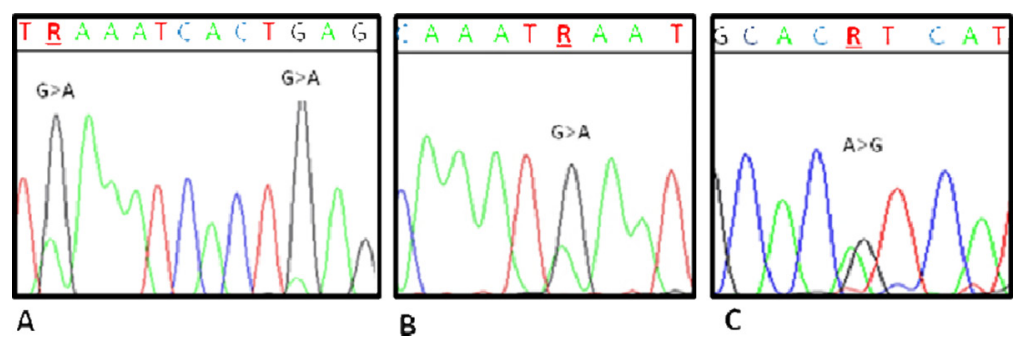

Figure 1: Electropherograms showing PIK3CA mutations. (A) Sample AQ03: PIK3CA exon 9 c.1624G $>$ A, E542K; c.1633G $>$ A, E545K. (B) Sample AQ02: PIK3CA exon 20 c.3129G>A, M1043I. (C) Sample AQ08: PIK3CA exon 20 c.3140A>G, H1047R. 


\begin{tabular}{|c|c|c|c|c|}
\hline & & $\begin{array}{c}\text { PIK3CA mutated } \\
\text { N }(\%)\end{array}$ & $\begin{array}{c}P I K 3 C A \text { wild-type } \\
\text { N }(\%)\end{array}$ & p value ${ }^{a}$ \\
\hline \multirow[t]{3}{*}{$\begin{array}{l}\mathrm{pCR} \text { versus Residual Disease } \\
\text { (nv } \rightarrow 3 \text { patients) }\end{array}$} & & & & 0.981 \\
\hline & $\mathrm{RD}$ & $9 / 11(82)$ & $23 / 28(82)$ & \\
\hline & $\mathrm{pCR}$ & 2/11 (18) & $5 / 28(18)$ & \\
\hline \multirow[t]{5}{*}{$\begin{array}{l}\text { Residual Cancer Burden } \\
\text { (nv } \rightarrow 8 \text { patients) }\end{array}$} & & & & 0.454 \\
\hline & 0 & $2 / 10(20)$ & $5 / 24(21)$ & \\
\hline & I & $1 / 10(10)$ & $2 / 24(8)$ & \\
\hline & II & $5 / 10(50)$ & $6 / 24(25)$ & \\
\hline & III & $2 / 10(20)$ & $11 / 24(46)$ & \\
\hline \multicolumn{5}{|l|}{$\mathrm{pCR}$} \\
\hline \multicolumn{5}{|l|}{ HER2+ } \\
\hline & $\mathrm{HR}+$ & $0 / 2$ & $1 / 8(12.5 \%)$ & 0.598 \\
\hline & HR- & $1 / 2(50 \%)$ & $3 / 9(33 \%)$ & 0.658 \\
\hline & Total & $1 / 4(25 \%)$ & $4 / 17(23.5 \%)$ & 0.950 \\
\hline \multicolumn{5}{|l|}{ HER2- } \\
\hline & $\mathrm{HR}+$ & $0 / 4$ & $0 / 8$ & nv \\
\hline & HR- & $1 / 3(33 \%)$ & $1 / 3(33 \%)$ & 1.000 \\
\hline & Total & $1 / 7(14 \%)$ & $1 / 11(9 \%)$ & 0.732 \\
\hline
\end{tabular}

${ }^{\text {a }}$ Chi-square test.

relapse rate among $\mathrm{TNBC}$ patients with residual disease [40]. In the HR-positive/HER2-negative population, we obtained a pCR rate of $7.7 \%$ if considering $\mathrm{EC} \rightarrow$ $\mathrm{D}$ and $7.1 \%$ if considering the sequential anthracycline and taxane chemotherapy. The $<10 \% \mathrm{pCR}$ rate in this subgroup, comparable with a meta-analytic pCR rate of $8.3 \%$ [37], imposes a better selection of patients, proposing neoadjuvant chemotherapy only to those with inoperable disease or candidate to adjuvant chemotherapy, for example for clinical node positive status, absent or low expression of PgR or high Ki67, which seem to influence the response to neoadjuvant treatment within the group of luminal tumors $[41,42]$. As to disease recurrences, we observed a higher rate of incidence in the triple-negative subgroup (67\%), which is the only one where median RFS, DFS and OS were reached: 12 months, 7.5 months and 29.5 months, respectively. Notably, among patients with TNBC, recurrences occurred even if ypN0 at surgery, as if nodal status at surgery did not influence long-term outcome of patients with this biologically aggressive disease. Conversely, among patients with HR+/HER2tumors, recurrences occurred only in $\mathrm{ypN}+$ patients at surgery, confirming the negative prognostic impact on survival of nodal residual disease after neoadjuvant chemotherapy [43].

In the present study, $P I K 3 C A$ mutational analysis did not define subgroups of patients most likely to achieve the pCR. Within the HER2-positive tumors, the prevalence of PIK3CA mutations (19\%) is comparable to results from neoadjuvant trials in the HER2-positive BC population, ranging from $19.2 \%$ to $24.3 \%[18,19,34]$. In this subgroup, we obtained a pCR rate of $25 \%$ in the mutated cohort and $23.5 \%$ in the wild-type. A pooled analysis of 967 patients from five prospective trials investigating double blockade with lapatinib and trastuzumab in the neoadjuvant setting, recently demonstrated that $P I K 3 C A$ mutations negatively influence pCR: overall, HER2positive tumors harboring mutations had significantly lower pCR rates compared to wild-type (16.2\% versus $29.6 \% ; \mathrm{p}<0.001)$. Anyway, a significantly different $\mathrm{pCR}$ rate between $P I K 3 C A$ mutated and wild-type tumors could only be observed in the group receiving trastuzumab and lapatinib $(16.7 \%$ versus $39.1 \%$; $<0.001)$, while only a trend toward worse response was observed in the cohort of patients treated with trastuzumab alone, whose pCR rate was $20.3 \%$ versus $27.1 \%$ for the mutated and wild- 
Table 7: Association between $P I K 3 C A$ genotype and long-term survival

\begin{tabular}{|c|c|c|c|}
\hline & \multicolumn{2}{|c|}{$\begin{array}{c}\text { Disease-Free Survival } \\
\text { (months and events/patients) }\end{array}$} & \multirow[t]{2}{*}{ p value ${ }^{a}$} \\
\hline & PIK3CA mutated & $P I K 3 C A$ wild-type & \\
\hline Overall & $\begin{array}{c}\mathbf{n r} \\
(4 / 11)\end{array}$ & $\begin{array}{c}\mathbf{5 2} \\
(12 / 28)\end{array}$ & 0.761 \\
\hline \multicolumn{4}{|l|}{ HER2+ } \\
\hline Overall & $\begin{array}{c}23 \\
(2 / 4)\end{array}$ & $\begin{array}{c}\mathbf{n r} \\
(5 / 17)\end{array}$ & 0.337 \\
\hline $\mathrm{HR}+$ & $\begin{array}{c}16 \\
(1 / 2)\end{array}$ & $\begin{array}{c}\mathbf{n r} \\
(3 / 8)\end{array}$ & 0.496 \\
\hline HR- & $\begin{array}{l}\mathbf{1 8 . 5} \\
(1 / 2)\end{array}$ & $\begin{array}{c}\mathbf{n r} \\
(2 / 9)\end{array}$ & 0.296 \\
\hline HR+/HER2- & $\begin{array}{c}\mathbf{n r} \\
(1 / 4)\end{array}$ & $\begin{array}{c}52 \\
(4 / 8)\end{array}$ & 0.640 \\
\hline TNBC & $\begin{array}{c}26 \\
(1 / 3)\end{array}$ & $\begin{array}{c}3 \\
(3 / 3)\end{array}$ & 0.115 \\
\hline
\end{tabular}

${ }^{\text {a }}$ Chi-square test.

type tumors, respectively (p 0.343) [44]. Consistently with our findings, an apparently lower pCR rate for $P I K 3 C A$ wild-type compared to mutated patients, in individuals treated with chemotherapy plus trastuzumab alone, was observed in the CHER-LOB study [45]. In this regard, a recent retrospective integrated analysis on HER2-positive patients treated with anthracycline-taxanebased chemotherapy plus trastuzumab as single blockade within the GeparQuattro study, PTEN and pEBP4, a PI3K downstream marker, added information to the predictive role of PIK3CA mutation status [46]. In line with results from the mentioned pooled analysis [44], an interaction between PIK3CA and HR status can be identified also in our analysis, even if not significant. HR-positive and PIK3CA mutated patients have the lowest incidence of pCR (any patient in this group achieved it), compared to HR-positive and PIK3CA wild-type (12.5\%), HRnegative and PIK3CA mutated (50\%) and HR-negative and PIK3CA wild-type (33\%) patients. Among patients with triple-negative and HR-positive/HER2-negative tumors, $P I K 3 C A$ mutational status did not affect the pCR rate. In the triple-negative subgroup, the pCR rate was $33 \%$ both for the mutated and the wild-type tumors, while in the HR-positive/HER2-negative subgroup any patients achieved pCR, neither wild-type nor mutated. To the best of our knowledge, to date no clinical studies have been performed to evaluate the role of PIK3CA mutations in the achievement of the pCR in these subgroups of patients. The activation of the PI3K pathway has been identified as an important issue in triple-negative/basal-like BC. As recently demonstrated, $P I K 3 C A$ kinase domain mutations can be frequently detected in androgen receptor (AR)- positive TNBC compared to the other subtypes (40\% versus 4\%) and targeting AR in preclinical study increases sensitivity to PI3K inhibitors [47]. An open label phase II randomized study, evaluating the addition of the mTOR inhibitor everolimus to a standard neoadjuvant chemotherapy for women with TNBC, did not result into a significant increase of pCR rate $(30.4 \%$ versus $25.9 \%$; p 0.76), even if a downregulation of the mTOR pathway at 48 hours in the everolimus arm was observed [48]. Regarding the HR-positive/HER2-negative disease in the neoadjuvant setting, there are no studies combining chemotherapy and inhibitors of the PI3K pathway. A phase II randomized trial was carried out to evaluate the rate of tumor response combining everolimus and letrozole in the neoadjuvant treatment of postmenopausal women with HR-positive BC. The response rates were $68.1 \%$ in the everolimus arm and $59.1 \%$ in the placebo arm ( $p$ 0.062), a difference considered statistically significant in the onesided $\chi 2$ test [49].

With respect to prognosis, our findings allow us to conclude that: a) patients with HER2-positive tumors tend to have a better long-term outcome in case of PIK3CA wild-type; b) patients with TNBC tend to have a better outcome in case of PIK3CA mutated; c) patients with HR-positive/HER2-negative tumors harboring PIK3CA mutation could probably still benefit from neoadjuvant chemotherapy since, although not achieving pCR, their median DFS has not yet been reached, compared to 52 months of patients with HR-positive/HER2-negative/ PIK3CA wild-type tumors. In the HER2-positive subgroup, the overall median DFS was not still reached for patients with $P I K 3 C A$ wild-type tumors, while it was 23 
months for the PIK3CA mutated ones. The difference was not statistically significant ( $\mathrm{p}$ 0.337), but a trend toward worse prognosis in case of mutation can be observed in the Kaplan-Meier curve (Figure 2). The prognostic effect of PIK3CA mutation within HER2-positive tumors is well established in the metastatic setting: in the CLEOPATRA trial, median PFS was longer for patients with wild-type PIK3CA in both the control and pertuzumab arms (8.6 months for mutated versus 13.8 months for wild-type in the control arm; 12.5 months for mutated versus 21.8 months for wild-type in the pertuzumab arm) [20]. On the other hand, in the neoadjuvant setting, only a trend toward an inferior OS for patients with a $P I K 3 C A$ mutated tumor was reported in the Gepar experience [18], but data on survival from the pooled analysis by Loibl et al [44] could not allow to draw definite conclusions. At a median followup of 47 months, there was no statistically significant difference in DFS between patients with wild-type and mutated tumors (hazard ratio mutated versus wild-type 1.07 ; p 0.691). However, a significant interaction between HR status and PIK3CA genotype was described. The
HR-positive subgroup showed a statistically significant longer DFS for the PIK3CA wild-type cohort (p 0.050), while in the HR-negative subgroup there was a nonsignificant trend for a longer DFS for the PIK3CA mutated

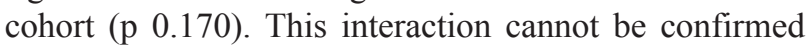
in our results, as we observed a better DFS for patients with wild-type tumors both in the HR-positive and in the HR-negative subgroups compared to mutated (16 months versus not reached for HR-positive tumors, respectively; 18.5 months versus not reached for HR-negative tumors, respectively). In the triple-negative subgroup, $P I K 3 C A$ mutations were associated with a worse long-term outcome: even if the difference was not statistically significant ( $\mathrm{p}$ 0.115), the overall median DFS was 3 months for patients with $P I K 3 C A$ wild-type tumors and 26 months for the $P I K 3 C A$ mutated ones. The presence of $P I K 3 C A$ mutations on cell-free DNA in plasma of patients with early-stage TNBC resulted as a positive prognostic factor on both RFS and BC-specific-survival [50]. A trend toward better OS for patients with TNBC harboring mutations in the PI3K pathway was also observed in a
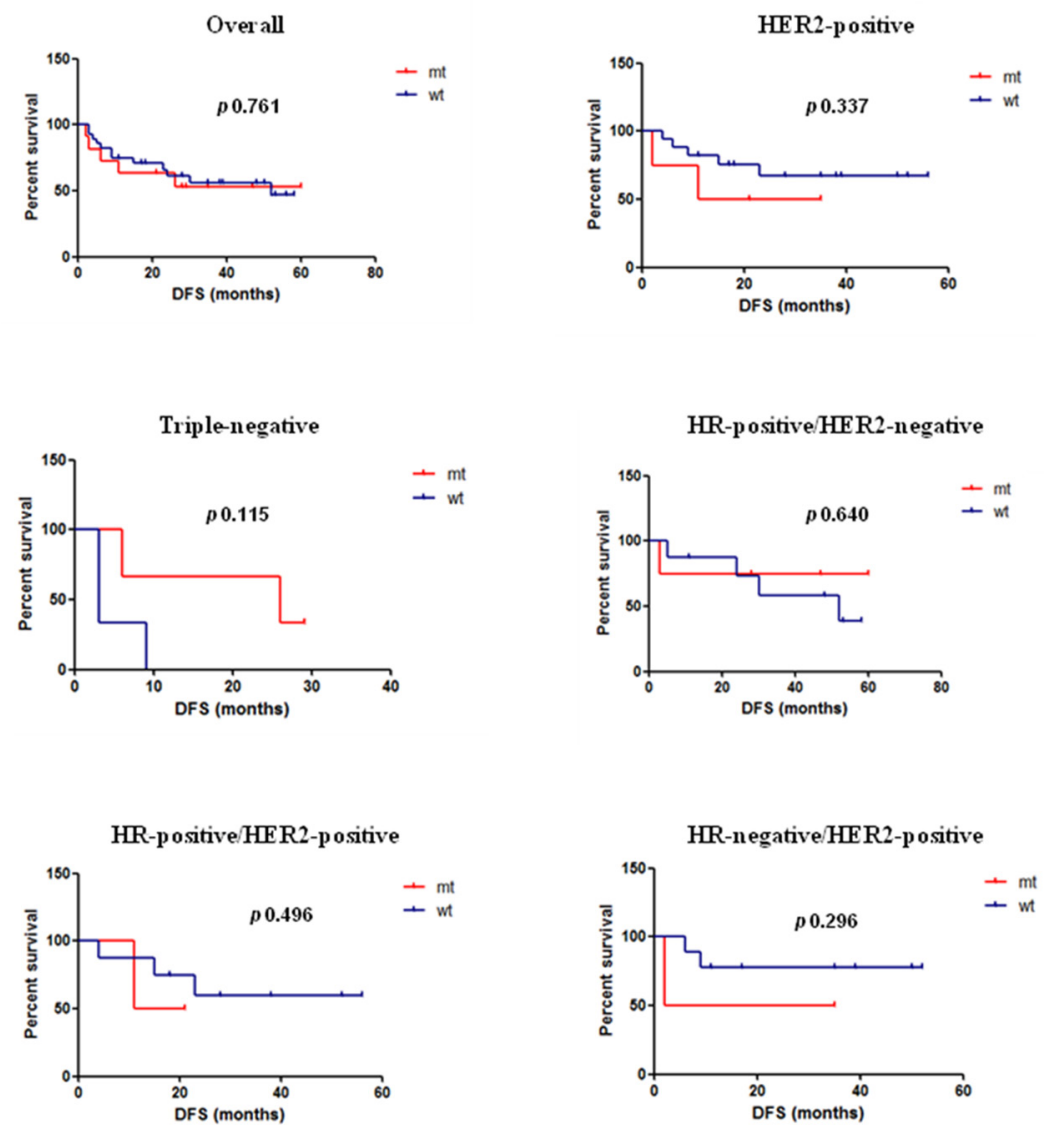

Figure 2: Kaplan-Meier curves of DFS overall and by subgroups according to PIK3CA genotype. Median DFS overall and by subgroups in the mutated versus wild-type cohort, respectively. Overall: not reached versus 52 months (p 0.761); HER2-positive: 23 months versus not reached (p 0.337); triple-negative: 26 months versus 3 months ( $\mathrm{p}$ 0.115); HR-positive/HER2-negative: not reached versus 52 months (p 0.640); HR-positive/HER2-positive: 16 months versus not reached (p 0.496); HR-negative/HER2-positive: 18.5 months versus not reached (p 0.296). 
cohort of 104 TNBC cases studied by whole exome sequencing in order to decipher the genetic landscape of these tumors and identify specific mutational patterns with prognostic potential [51]. In the HR-positive/HER2negative subgroup, patients with PIK3CA mutated tumors seem to have a longer DFS (not yet reached) compared to wild-type. Any patients in this subgroup achieved the $\mathrm{pCR}$, but it could be hypothesized that, beside the clinicopathological parameters mentioned above (nodal status, PgR expression, Ki67) also PIK3CA mutation status can be considered a useful variable to indicate a chemotherapy to affected patients. Larger studies may help answer this question.

The present study has some substantial limitations, mainly related to the small number of patients in each subgroup, affecting the power of statistical analysis and results. Anyway, the strength of this work lies in the analysis of a homogeneous series of patients, all treated with the same chemotherapy regimen, and in the strictness of the PIK3CA mutational analysis, making it an interesting and well-done example of translational research in real-life clinical practice.

\section{MATERIALS AND METHODS}

\section{Inclusion criteria and patients' evaluation}

Women with invasive, previously untreated, clinical stage II/III or inflammatory BC, histologically confirmed from a core biopsy specimen, aging 18-75, World Health Organization performance status $\leq 2$, adequate cardiac (echocardiographic LVEF $\geq 50 \%$ ), haematological, hepatic and renal functions were enrolled. Exclusion criteria included uncontrolled severe comorbidities, particularly ischemic heart disease (myocardial infarction, angina pectoris), arrhythmia, New York Heart Association (NYHA) grade $\geq$ II or congestive heart failure (CHF).

$\mathrm{BC}$ patients were treated in clinical practice, based on the indication of epirubicin, cyclophosphamide, docetaxel and trastuzumab approved by Agenzia Italiana del Farmaco (AIFA) for administration in label in Italian public hospitals and on the evaluation of the Institutional Review Board of San Salvatore Hospital, L'Aquila, Italy. All patients gave written informed consent to participate. The protocol was conducted in accordance with the Declaration of Helsinki of the World Medical Association and Good Clinical Practice.

Before every treatment cycle administration, patients underwent clinical examination and assessment of blood chemistry, toxic effects and tumor size. Toxicity was evaluated according to National Cancer Institute Common Toxicity Criteria (NCI-CTC, version 4.02). Cardiac monitoring was conducted by ECG and biochemical monitoring (blood dosage of pBNP and troponin I) at d1 of every cycle and Echocardiogram with LVEF evaluation at baseline, after the EC phase and at treatment completion.

\section{Study design and treatment plan}

This was a single-arm, single-centre, phase I-II study of dose-dense (every 2 weeks) docetaxel (D) (starting dose $65 \mathrm{mg} / \mathrm{m}^{2}$, that is the $\mathrm{RD}$ reached in combination with non-pegylated liposomal doxorubicin in a dosedense schedule, as we recently reported [52]), combined with trastuzumab (T) $(4 \mathrm{mg} / \mathrm{kg}$, loading dose $6 \mathrm{mg} / \mathrm{kg})$ in HER2-positive patients, for 4 cycles following dosedense epirubicin plus cyclophosphamide (EC) $(90 / 600$ $\mathrm{mg} / \mathrm{m}^{2}$ ) for 4 cycles, as neoadjuvant chemotherapy in patients with BC. An inter-patient dose-de-escalation approach was planned, scheduling dose reduction to 60 , 55 and $50 \mathrm{mg} / \mathrm{m}^{2}$ [53]. Trastuzumab dose was adapted to the biweekly administration of chemotherapy, based on the demonstrated schedule independence of its linear pharmacokinetics [54], to maintain a dose intensity of 2 $\mathrm{mg} / \mathrm{kg} / \mathrm{w}$. Pegylated G-CSF was scheduled 24 hours after chemotherapy administration.

Dose-limiting toxicities (DLTs) were defined as G4 haematological; G3 non-haematological; any toxicity resulting in $>2$ weeks chemotherapy delay; $\geq 10 \%$ LVEF reduction from baseline, if final value was $<50 \%$ or $\geq 20 \%$ LVEF reduction from baseline if final value was $\geq 50 \%$, arrhythmia, symptomatic heart failure. Definitive surgery, either breast conservation or mastectomy with complete axillary lymph node dissection, was performed at treatment completion and recommended three weeks after chemotherapy discontinuation.

Primary end-points were: determination of $\mathrm{D} \mathrm{RD}$, in combination with $\mathrm{T}$ in HER2-positive patients, in a dosedense schedule following dose-dense EC combination (phase I); the activity (measured by the pCR rate, defined as absence of invasive residual $\mathrm{BC}$ in the breast and axillary lymph nodes in all surgically excised specimens) and efficacy (measured by RFS, DFS and OS) (phase II) of the proposed regimen. Secondary end points included: the assessment of the correlation between activity and efficacy of treatment with the PIK3CA mutation status before chemotherapy, safety and compliance.

\section{Immunohistochemical and pathologic assessment}

ER, PgR and HER2 analysis was performed on pretreatment core needle biopsy specimens using immunohistochemical staining techniques. ER and PgR positivity was defined as staining in $\geq 10 \%$ of cells. HER2 analysis was done using the HercepTest (Dako), with staining intensity score evaluated from 0 to $3+$. For specimens staining 2+, Fluorescent in situ Hybridization analysis was performed to assess HER2 amplification (ratio $>2.2$ ).

To overcome the simplistic dichotomization of response in terms of $\mathrm{pCR}$ or residual disease, the latter including a broad range of responses, the RCB was measured for non-pCR tumors, calculated as a continuous 
variable derived from the primary tumor dimensions, cellularity of the tumor bed and axillary nodal burden [55].

\section{PIK3CA mutation assessment}

Contiguous sections $3 \mu \mathrm{m}$ thick from the interior of a formalin-fixed paraffin-embedded (FFPE) tissue block were cut. Hematoxilyn and eosin staining was performed to assess the presence and amount of tumor cells. Genomic DNA was extracted (FFPE DNA purification kit, Norgen Biotek Corp.), by following the manufacturer's instructions. To detect hot-spot PI3KCA mutations, polymerase chain reaction (40 cycles) was performed by using $50 \mathrm{ng}$ genomic DNA, dNTPs $250 \mu \mathrm{M}, \mathrm{MgCl}_{2}$ $1.5 \mathrm{mM}$, Taq DNA polymerase (Invitrogen) $5 \mathrm{U}$, primers $10 \mathrm{pmol}$ each (as reported by Sueta et al [56]). Amplified products were purified (Qiaquick PCR purification Kit, Qiagen). Thirty nanograms of purified PCR products were subjected to sequencing by using the Big Dye terminator v3.1 sequencing kit (Applied Biosystems) according to the manufacturer's instructions and run on a 3500 Genetic Analyzer (Applied Biosystems).

\section{Statistical analysis}

The sample size was calculated according to the Simon's two-stage phase II optimal design. For the HER2positive and triple-negative tumors, the null response rate $\left(p_{0}\right)$ below which there would be no further interest in the proposed regimen was set at $20 \%$, and the rate beyond which further studies would be of interest $\left(p_{l}\right)$ was set at $40 \%$. Assuming an $\alpha$ error rate of 0.05 and a $\beta$ error rate of $0.20,13$ patients were to be accrued in the first step. If $\geq 3$ pCRs were observed, 30 more patients were to be enrolled. The regimen was considered of clinical interest if $\geq 12$ pCRs were observed among 43 patients. For the HRpositive/HER2-negative tumors, $p_{0}$ was set at $10 \%$ and $p_{i}$ was set at $30 \%$. Assuming an $\alpha$ error rate of 0.05 and a $\beta$ error rate of $0.20,10$ patients were to be accrued in the first step. If $\geq 1 \mathrm{pCRs}$ were observed, 19 more patients were to be enrolled. The regimen was considered of clinical interest if $\geq 5$ pCRs were observed among 29 patients. RFS, DFS OS were evaluated by Kaplan-Meier method [57]. RFS was evaluated from the beginning of treatment to disease recurrence or last contact; DFS from surgery to disease recurrence or last contact; OS from the beginning of treatment to death or last contact. The significance of differences between survival rates was evaluated with the log-rank test. Relationships between PIK3CA mutation status and clinical, histological and biological parameters were estimated with the chi-squared test.

\section{Abbreviations}

PI3K: phosphatidylinositol-3-kinase; mTOR: mammalian target of rapamycin; BC: breast cancer; HER2: human epidermal growth factor receptor 2; ER: estrogen receptor; pCR: pathological complete response; DFS: disease-free survival; OS: overall survival; HR: hormone receptor; dd: dose-dense; RFS: relapse-free survival; T: trastuzumab; RD: recommended dose; D: Docetaxel; EC: Epirubicin/Cyclophosphamide; LABC: locally advanced $\mathrm{BC}$; IBC: inflammatory breast cancer; PgR: Progesterone Receptor; DLT: Dose-limiting toxicity; HFS: hand-foot syndrome; nab: nanoparticle-albumin-bound; DI: dose intensity; ITT: intention-to-treat; TNBC: triple negative breast cancer; $p B N P$ : precursor-Brain-Natriuretic-Peptide; LVEF: left ventricular ejection fraction; RCB: Residual Cancer Burden; CHF: congestive heart failure; FFPE: formalin-fixed paraffin-embedded; OR: odds ratio; CI: Confidence Interval; AR: androgen receptor.

\section{Author contributions}

Conception and design: Katia Cannita, Valentina Cocciolone, Alessandra Tessitore; Development of methodology: Katia Cannita, Valentina Cocciolone, Alessandra Tessitore; Provision of study materials or patients: Katia Cannita, Valentina Cocciolone, Alessandra Tessitore, Valentina Mastroiaco, Lucia Rinaldi, Stefania Paradisi, Azzurra Irelli, Paola Lanfiuti Baldi, Tina Sidoni, Enrico Ricevuto, Antonella Dal Mas, Giuseppe Calvisi, Gino Coletti, Antonietta Ciccozzi, Laura Pizzorno, Valter Resta, Alberto Bafile, Edoardo Alesse, Corrado Ficorella; Acquisition of data: Valentina Cocciolone, Valentina Mastroiaco, Alessandra Tessitore, Katia Cannita; Analysis and interpretation of data: Valentina Cocciolone, Katia Cannita, Alessandra Tessitore, Valentina Mastroiaco; Writing and revision of the manuscript: Valentina Cocciolone, Alessandra Tessitore, Katia Cannita, Corrado Ficorella; Final approval of manuscript: All authors.

\section{ACKNOWLEDGMENTS}

We thank Dr. Virginia Ciuffetelli, Pathology Department, S. Salvatore Hospital, L'Aquila, Italy, for contribution in collection of biological materials.

\section{CONFLICTS OF INTEREST}

The authors declare that they have no competing interests.

\section{REFERENCES}

1. Mauri D, Pavlidis N, Ioannidis JP. Neoadjuvant versus adjuvant systemic treatment in breast cancer: a metaanalysis. J Natl Cancer Inst. 2005; 97:188-94.

2. Fumagalli D, Bedard PL, Nahleh Z, Michiels S, Sotiriou C, Loi S, Sparano JA, Ellis M, Hylton N, Zujewski JA, Hudis C, Esserman L, Piccart M, and BIG-NABCG collaboration. A common language in neoadjuvant breast cancer clinical 
trials: proposals for standard definitions and endpoints. Lancet Oncol. 2012; 13:e240-48.

3. van der Hage JA, van de Velde CJ, Julien JP, TubianaHulin M, Vandervelden C, Duchateau L. Preoperative chemotherapy in primary operable breast cancer: results from the European Organization for Research and Treatment of Cancer trial 10902. J Clin Oncol. 2001; 19:4224-37.

4. Scholl SM, Pierga JY, Asselain B, Beuzeboc P, Dorval T, Garcia-Giralt E, Jouve M, Palangié T, Remvikos Y, Durand JC, Fourquet A, Pouillart P. Breast tumour response to primary chemotherapy predicts local and distant control as well as survival. Eur J Cancer. 1995; 31A:1969-75.

5. Kuerer HM, Newman LA, Smith TL, Ames FC, Hunt KK, Dhingra K, Theriault RL, Singh G, Binkley SM, Sneige N, Buchholz TA, Ross MI, McNeese MD, et al. Clinical course of breast cancer patients with complete pathologic primary tumor and axillary lymph node response to doxorubicinbased neoadjuvant chemotherapy. J Clin Oncol. 1999; 17:460-69.

6. Cortazar P, Zhang L, Untch M, Mehta K, Costantino JP, Wolmark N, Bonnefoi H, Cameron D, Gianni L, Valagussa P, Swain SM, Prowell T, Loibl S, et al. Pathological complete response and long-term clinical benefit in breast cancer: the CTNeoBC pooled analysis. Lancet. 2014; 384:164-72. https://doi.org/10.1016/ S0140-6736(13)62422-8.

7. Buzdar AU, Ibrahim NK, Francis D, Booser DJ, Thomas ES, Theriault RL, Pusztai L, Green MC, Arun BK, Giordano SH, Cristofanilli M, Frye DK, Smith TL, et al. Significantly higher pathologic complete remission rate after neoadjuvant therapy with trastuzumab, paclitaxel, and epirubicin chemotherapy: results of a randomized trial in human epidermal growth factor receptor 2-positive operable breast cancer. J Clin Oncol. 2005; 23:3676-85.

8. Gianni L, Eiermann W, Semiglazov V, Manikhas A, Lluch A, Tjulandin S, Zambetti M, Vazquez F, Byakhow M, Lichinitser M, Climent MA, Ciruelos E, Ojeda B, et al. Neoadjuvant chemotherapy with trastuzumab followed by adjuvant trastuzumab versus neoadjuvant chemotherapy alone, in patients with HER2-positive locally advanced breast cancer (the NOAH trial): a randomised controlled superiority trial with a parallel HER2-negative cohort. Lancet. 2010; 375:377-84.

9. Gianni L, Eiermann W, Semiglazov V, Lluch A, Tjulandin S, Zambetti M, Moliterni A, Vazquez F, Byakhov MJ, Lichinitser M, Climent MA, Ciruelos E, Ojeda B, et al. Neoadjuvant and adjuvant trastuzumab in patients with HER2-positive locally advanced breast cancer (NOAH): follow-up of a randomised controlled superiority trial with a parallel HER2-negative cohort. Lancet Oncol. 2014; 15:640-47.

10. Norton L. Evolving concepts in the systemic drug therapy of breast cancer. Semin Oncol. 1997; 24:S10-3-S10-10.
11. Citron ML, Berry DA, Cirrincione C, Hudis C, Winer EP, Gradishar WJ, Davidson NE, Martino S, Livingston R, Ingle JN, Perez EA, Carpenter J, Hurd D, et al. Randomized trial of dose-dense versus conventionally scheduled and sequential versus concurrent combination chemotherapy as postoperative adjuvant treatment of node-positive primary breast cancer: first report of Intergroup Trial C9741/Cancer and Leukemia Group B Trial 9741. J Clin Oncol. 2003; 21:1431-39.

12. Lin NU, Gelman R, Winer EP. Dose density in breast cancer: a simple message? J Natl Cancer Inst. 2005; 97:1712-14.

13. Bonilla L, Ben-Aharon I, Vidal L, Gafter-Gvili A, Leibovici L, Stemmer SM. Dose-dense chemotherapy in nonmetastatic breast cancer: a systematic review and metaanalysis of randomized controlled trials. J Natl Cancer Inst. 2010; 102:1845-54.

14. Loi S, Michiels S, Lambrechts D, Fumagalli D, Claes B, Kellokumpu-Lehtinen PL, Bono P, Kataja V, Piccart MJ, Joensuu H, Sotiriou C. Somatic mutation profiling and associations with prognosis and trastuzumab benefit in early breast cancer. J Natl Cancer Inst. 2013; 105:960-67.

15. Stemke-Hale K, Gonzalez-Angulo AM, Lluch A, Neve RM, Kuo WL, Davies M, Carey M, Hu Z, Guan Y, Sahin A, Symmans WF, Pusztai L, Nolden LK, et al. An integrative genomic and proteomic analysis of PIK3CA, PTEN, and AKT mutations in breast cancer. Cancer Res. 2008; 68:6084-91.

16. Cancer Genome Atlas Network. Comprehensive molecular portraits of human breast tumours. Nature. 2012; 490:61-70.

17. Liedtke C, Cardone L, Tordai A, Yan K, Gomez HL, Figureoa LJ, Hubbard RE, Valero V, Souchon EA, Symmans WF, Hortobagyi GN, Bardelli A, Pusztai L. PIK3CAactivating mutations and chemotherapy sensitivity in stage II-III breast cancer. Breast Cancer Res. 2008; 10:R27.

18. Loibl S, von Minckwitz G, Schneeweiss A, Paepke S, Lehmann A, Rezai M, Zahm DM, Sinn P, Khandan F, Eidtmann H, Dohnal K, Heinrichs C, Huober J, et al. PIK3CA mutations are associated with lower rates of pathologic complete response to anti-human epidermal growth factor receptor 2 (her2) therapy in primary HER2-overexpressing breast cancer. J Clin Oncol. 2014; 32:3212-20.

19. Majewski IJ, Nuciforo P, Mittempergher L, Bosma AJ, Eidtmann H, Holmes E, Sotiriou C, Fumagalli D, Jimenez J, Aura C, Prudkin L, Díaz-Delgado MC, de la Peña L, et al. PIK3CA mutations are associated with decreased benefit to neoadjuvant human epidermal growth factor receptor 2-targeted therapies in breast cancer. J Clin Oncol. 2015; 33:1334-39.

20. Baselga J, Cortés J, Im SA, Clark E, Ross G, Kiermaier A, Swain SM. Biomarker analyses in CLEOPATRA: a phase 
III, placebo-controlled study of pertuzumab in human epidermal growth factor receptor 2-positive, first-line metastatic breast cancer. J Clin Oncol. 2014; 32:3753-61.

21. Miller KD, McCaskill-Stevens W, Sisk J, Loesch DM, Monaco F, Seshadri R, Sledge GW Jr. Combination versus sequential doxorubicin and docetaxel as primary chemotherapy for breast cancer: A randomized pilot trial of the Hoosier Oncology Group. J Clin Oncol. 1999; 17:3033-37.

22. Cooper BW, Radivoyevitch T, Overmoyer BA, Shenk RR, Pham HT, Samuels JR, Parry MP, Silverman P. Phase II study of dose-dense sequential doxorubicin and docetaxel for patients with advanced operable and inoperable breast cancer. Breast Cancer Res Treat. 2006; 97:311-18.

23. Antolín S, Mel R, Ramos M, García-Palomo A, Almanza C, de Paz L, Calvo L, Alvarez E, González A, García-Mata J. A dose-dense schedule of docetaxel followed by doxorubicin and cyclophosphamide as neoadjuvant treatment for breast cancer: results from a phase II study. Clin Transl Oncol. 2011; 13:686-91.

24. Natoli C, Cianchetti E, Tinari N, Angelucci D, Grassadonia A, Zilli M, Ficorella C, Ricevuto E, Grossi S, De Tursi M, Carella C, Rispoli AI, Iacobelli S. A phase II study of dose-dense epirubicin plus cyclophosphamide followed by docetaxel plus capecitabine and pegfilgrastim support as preoperative therapy for patients with stage II, IIIA breast cancer. Ann Oncol. 2007; 18:1015-20.

25. Bear HD, Anderson S, Brown A, Smith R, Mamounas EP, Fisher B, Margolese R, Theoret H, Soran A, Wickerham DL, Wolmark N, and National Surgical Adjuvant Breast and Bowel Project Protocol B-27. The effect on tumor response of adding sequential preoperative docetaxel to preoperative doxorubicin and cyclophosphamide: preliminary results from National Surgical Adjuvant Breast and Bowel Project Protocol B-27. J Clin Oncol. 2003; 21:4165-74.

26. Valachis A, Mauri D, Polyzos NP, Chlouverakis G, Mavroudis D, Georgoulias V. Trastuzumab combined to neoadjuvant chemotherapy in patients with HER2-positive breast cancer: a systematic review and meta-analysis. Breast. 2011; 20:485-90.

27. Pierga JY, Delaloge S, Espié M, Brain E, Sigal-Zafrani B, Mathieu MC, Bertheau P, Guinebretière JM, Spielmann M, Savignoni A, Marty M. A multicenter randomized phase II study of sequential epirubicin/cyclophosphamide followed by docetaxel with or without celecoxib or trastuzumab according to HER2 status, as primary chemotherapy for localized invasive breast cancer patients. Breast Cancer Res Treat. 2010; 122:429-37.

28. Untch M, Fasching PA, Konecny GE, Hasmüller S, Lebeau A, Kreienberg R, Camara O, Müller V, du Bois A, Kühn T, Stickeler E, Harbeck N, Höss C, et al. Pathologic complete response after neoadjuvant chemotherapy plus trastuzumab predicts favorable survival in human epidermal growth factor receptor 2-overexpressing breast cancer: results from the TECHNO trial of the AGO and GBG study groups. J Clin Oncol. 2011; 29:3351-57.

29. Buzdar AU, Suman VJ, Meric-Bernstam F, Leitch AM, Ellis MJ, Boughey JC, Unzeitig G, Royce M, McCall LM, Ewer MS, Hunt KK, and American College of Surgeons Oncology Group investigators. Fluorouracil, epirubicin, and cyclophosphamide (FEC-75) followed by paclitaxel plus trastuzumab versus paclitaxel plus trastuzumab followed by FEC-75 plus trastuzumab as neoadjuvant treatment for patients with HER2-positive breast cancer (Z1041): a randomised, controlled, phase 3 trial. Lancet Oncol. 2013; 14:1317-25.

30. Baselga J, Bradbury I, Eidtmann H, Di Cosimo S, de Azambuja E, Aura C, Gómez H, Dinh P, Fauria K, Van Dooren V, Aktan G, Goldhirsch A, Chang TW, et al, and NeoALTTO Study Team. Lapatinib with trastuzumab for HER2-positive early breast cancer (NeoALTTO): a randomised, open-label, multicentre, phase 3 trial. Lancet. 2012; 379:633-40.

31. Gianni L, Pienkowski T, Im YH, Roman L, Tseng LM, Liu MC, Lluch A, Staroslawska E, de la Haba-Rodriguez J, Im SA, Pedrini JL, Poirier B, Morandi P, et al. Efficacy and safety of neoadjuvant pertuzumab and trastuzumab in women with locally advanced, inflammatory, or early HER2-positive breast cancer (NeoSphere): a randomised multicentre, open-label, phase 2 trial. Lancet Oncol. 2012; 13:25-32.

32. Robidoux A, Tang G, Rastogi P, Geyer CE Jr, Azar CA, Atkins JN, Fehrenbacher L, Bear HD, Baez-Diaz L, Sarwar S, Margolese RG, Farrar WB, Brufsky AM, et al. Lapatinib as a component of neoadjuvant therapy for HER2-positive operable breast cancer (NSABP protocol B-41): an openlabel, randomised phase 3 trial. Lancet Oncol. 2013; 14:1183-92.

33. Schneeweiss A, Chia S, Hickish T, Harvey V, Eniu A, Hegg R, Tausch C, Seo JH, Tsai YF, Ratnayake J, McNally V, Ross G, Cortés J. Pertuzumab plus trastuzumab in combination with standard neoadjuvant anthracyclinecontaining and anthracycline-free chemotherapy regimens in patients with HER2-positive early breast cancer: a randomized phase II cardiac safety study (TRYPHAENA). Ann Oncol. 2013; 24:2278-84.

34. Guarneri V, Frassoldati A, Bottini A, Cagossi K, Bisagni G, Sarti S, Ravaioli A, Cavanna L, Giardina G, Musolino A, Untch M, Orlando L, Artioli F, et al. Preoperative chemotherapy plus trastuzumab, lapatinib, or both in human epidermal growth factor receptor 2-positive operable breast cancer: results of the randomized phase II CHER-LOB study. J Clin Oncol. 2012; 30:1989-95.

35. von Minckwitz G, Raab G, Caputo A, Schütte M, Hilfrich J, Blohmer JU, Gerber B, Costa SD, Merkle E, Eidtmann H, Lampe D, Jackisch C, du Bois A, Kaufmann M. Doxorubicin with cyclophosphamide followed by docetaxel every 21 days compared with doxorubicin and docetaxel 
every 14 days as preoperative treatment in operable breast cancer: the GEPARDUO study of the German Breast Group. J Clin Oncol. 2005; 23:2676-85.

36. Gerber B, Loibl S, Eidtmann H, Rezai M, Fasching PA, Tesch H, Eggemann H, Schrader I, Kittel K, Hanusch C, Kreienberg R, Solbach C, Jackisch C, et al, and German Breast Group Investigators. Neoadjuvant bevacizumab and anthracycline-taxane-based chemotherapy in 678 triple-negative primary breast cancers; results from the geparquinto study (GBG 44). Ann Oncol. 2013; 24:2978-84.

37. Houssami N, Macaskill P, von Minckwitz G, Marinovich ML, Mamounas E. Meta-analysis of the association of breast cancer subtype and pathologic complete response to neoadjuvant chemotherapy. Eur J Cancer. 2012; 48:3342-54.

38. Gluz O, Nitz UA, Harbeck N, Ting E, Kates R, Herr A, Lindemann W, Jackisch C, Berdel WE, Kirchner H, Metzner B, Werner F, Schütt G, et al, and West German Study Group. Triple-negative high-risk breast cancer derives particular benefit from dose intensification of adjuvant chemotherapy: results of WSG AM-01 trial. Ann Oncol. 2008; 19:861-70.

39. Lehmann BD, Bauer JA, Chen X, Sanders ME, Chakravarthy AB, Shyr Y, Pietenpol JA. Identification of human triple-negative breast cancer subtypes and preclinical models for selection of targeted therapies. J Clin Invest. 2011; 121:2750-67.

40. Carey LA, Dees EC, Sawyer L, Gatti L, Moore DT, Collichio F, Ollila DW, Sartor CI, Graham ML, Perou CM. The triple negative paradox: primary tumor chemosensitivity of breast cancer subtypes. Clin Cancer Res. 2007; 13:2329-34.

41. Ingolf JB, Russalina M, Simona M, Julia R, Gilda S, Bohle RM, Andrea H, Erich S, Daniel H. Can ki-67 play a role in prediction of breast cancer patients' response to neoadjuvant chemotherapy? BioMed Res Int. 2014; 2014:628217.

42. Colleoni M, Bagnardi V, Rotmensz N, Viale G, Mastropasqua M, Veronesi P, Cardillo A, Torrisi R, Luini A, Goldhirsch A. A nomogram based on the expression of Ki-67, steroid hormone receptors status and number of chemotherapy courses to predict pathological complete remission after preoperative chemotherapy for breast cancer. Eur J Cancer. 2010; 46:2216-24.

43. Zhang GC, Zhang YF, Xu FP, Qian XK, Guo ZB, Ren CY, Yao M. Axillary lymph node status, adjusted for pathologic complete response in breast and axilla after neoadjuvant chemotherapy, predicts differential disease-free survival in breast cancer. Curr Oncol. 2013; 20:e180-92.

44. Loibl S, Majewski I, Guarneri V, Nekljudova V, Holmes E, Bria E, Denkert C, Schem C, Sotiriou C, Loi S, Untch M, Conte P, Bernards R, et al. PIK3CA mutations are associated with reduced pathological complete response rates in primary HER2-positive breast cancer: pooled analysis of 967 patients from five prospective trials investigating lapatinib and trastuzumab. Ann Oncol. 2016; 27:1519-25.

45. Guarneri V, Dieci MV, Frassoldati A, Maiorana A, Ficarra G, Bettelli S, Tagliafico E, Bicciato S, Generali DG, Cagossi K, Bisagni G, Sarti S, Musolino A, et al. Prospective Biomarker Analysis of the Randomized CHERLOB Study Evaluating the Dual Anti-HER2 Treatment With Trastuzumab and Lapatinib Plus Chemotherapy as Neoadjuvant Therapy for HER2-Positive Breast Cancer. Oncologist. 2015; 20:1001-10.

46. Loibl S, Darb-Esfahani S, Huober J, Klimowicz A, Furlanetto J, Lederer B, Hartmann A, Eidtmann H, Pfitzner B, Fasching PA, Tiemann K, Jackisch C, Mehta $\mathrm{K}$, et al. Integrated Analysis of PTEN and p4EBP1 Protein Expression as Predictors for pCR in HER2-Positive Breast Cancer. Clin Cancer Res. 2016; 22:2675-83.

47. Lehmann BD, Bauer JA, Schafer JM, Pendleton CS, Tang L, Johnson KC, Chen X, Balko JM, Gómez H, Arteaga CL, Mills GB, Sanders ME, Pietenpol JA. PIK3CA mutations in androgen receptor-positive triple negative breast cancer confer sensitivity to the combination of PI3K and androgen receptor inhibitors. Breast Cancer Res. 2014; 16:406.

48. Gonzalez-Angulo AM, Akcakanat A, Liu S, Green MC, Murray JL, Chen H, Palla SL, Koenig KB, Brewster AM, Valero V, Ibrahim NK, Moulder-Thompson S, Litton JK, et al. Open-label randomized clinical trial of standard neoadjuvant chemotherapy with paclitaxel followed by FEC versus the combination of paclitaxel and everolimus followed by FEC in women with triple receptor-negative breast cancer. Ann Oncol. 2014; 25:1122-27.

49. Baselga J, Semiglazov V, van Dam P, Manikhas A, Bellet M, Mayordomo J, Campone M, Kubista E, Greil R, Bianchi G, Steinseifer J, Molloy B, Tokaji E, et al. Phase II randomized study of neoadjuvant everolimus plus letrozole compared with placebo plus letrozole in patients with estrogen receptor-positive breast cancer. J Clin Oncol. 2009; 27:2630-37.

50. Takeshita T, Yamamoto Y, Yamamoto-Ibusuki M, Inao T, Sueta A, Fujiwara S, Omoto Y, Iwase H. Prognostic role of PIK3CA mutations of cell-free DNA in early-stage triple negative breast cancer. Cancer Sci. 2015; 106:1582-89.

51. Kriegsmann M, Endris V, Wolf T, Pfarr N, Stenzinger A, Loibl S, Denkert C, Schneeweiss A, Budczies J, Sinn P, Weichert W. Mutational profiles in triple-negative breast cancer defined by ultradeep multigene sequencing show high rates of PI3K pathway alterations and clinically relevant entity subgroup specific differences. Oncotarget. 2014; 5:9952-65. https://doi.org/10.18632/oncotarget.2481.

52. Ricevuto E, Cocciolone V, Mancini M, Cannita K, Romano S, Bruera G, Pelliccione M, Adinolfi MI, Ciccozzi A, Bafile A, Penco M, Ficorella C. Dose-dense nonpegylated liposomal Doxorubicin and docetaxel combination in breast cancer: dose-finding study. Oncologist. 2015; 20:109-10. 
53. Storer BE. Design and analysis of phase I clinical trials. Biometrics. 1989; 45:925-37.

54. Bruno R, Washington CB, Lu JF, Lieberman G, Banken L, Klein P. Population pharmacokinetics of trastuzumab in patients with HER2+ metastatic breast cancer. Cancer Chemother Pharmacol. 2005; 56:361-69.

55. Symmans WF, Peintinger F, Hatzis C, Rajan R, Kuerer H, Valero V, Assad L, Poniecka A, Hennessy B, Green M, Buzdar AU, Singletary SE, Hortobagyi GN, Pusztai L. Measurement of residual breast cancer burden to predict survival after neoadjuvant chemotherapy. J Clin Oncol. 2007; 25:4414-22.

56. Sueta A, Yamamoto Y, Yamamoto-Ibusuki M, Hayashi M, Takeshita T, Yamamoto S, Iwase H. An integrative analysis of PIK3CA mutation, PTEN, and INPP4B expression in terms of trastuzumab efficacy in HER2-positive breast cancer. PLoS One. 2014; 9:e116054.

57. Kaplan EL, Meier P. Nonparametric estimation of incomplete observations. J Am Stat Assoc. 1958; $53: 457-81$. 